\title{
ALMOST CONTINUITY IMPLIES CLOSURE CONTINUITY ${ }^{\dagger}$
}

\author{
by MOHAMMAD SALEH
}

(Received 5 November, 1996)

\begin{abstract}
The purpose of this note is to answer in the affirmative a long standing open question raised by Singal and Singal-whether every almost continuous function is closure continuous ( $\theta$-continuous).
\end{abstract}

Introduction. Among other generalizations of continuity, the concepts of weak and closure continuity have been studied by many mathematicians: D. R. Andrew, J. Chew, L. Herrington, N. Levine, P. E. Long, T. Noire, J. Porter, M. Saleh, J. Tong, E. K. Whittlesy and others. In 1961, Levine introduced the concept of weak continuity as a generalization of continuity: later in 1966, Andrew and Whittlesy [2] introduced the concept of closure continuity which is stronger than weak continuity. Indeed closure continuity was introduced many years earlier by S. Fomin [3] precisely in 1941 as $\theta$-continuity, but it seems that Andrew and Whittlesy were not aware of the paper by Fomin. In 1968, Singal and Singal [9] introduced almost continuity as another generalization and raised the following question in Remark 3.3: is every almost continuous function $\theta$-continuous? In this short note we answer this question positively.

Definitions and notation. Let $A$ be a subset of a topological space $X$. The closure and the interior of $A$ in $X$ are denoted, respectively, by $\bar{A}, A^{\circ}$. A function $f: X \rightarrow Y$ is closure continuous ( $\theta$-continuous) at $x \in X$ if, given any open set $V$ in $Y$ containing $f(x)$, there exists an open set $U$ in $X$ containing $x$ such that $f(\bar{U}) \subseteq \bar{V}$. If this condition is satisfied at each $x \in X$, then $f$ is said to be closure continuous ( $\theta$-continuous). A function $f: X \rightarrow Y$ is said to be almost continuous in the sense of Singal and Singal if for each point $x \in X$ and each open set $V$ in $Y$ containing $f(x)$, there exists an open set $U$ in $X$ containing $x$ such that $f(U) \subseteq \bar{V}^{\circ}$.

Theorem 1. Let $f: X \rightarrow Y$ be almost continuous. Then $f$ is closure continuous.

Proof. Let $x \in X$ and let $V$ be an open set containing $f(x)$. By almost continuity of $f$, there exists an open set $U$ containing $x$ such that $f(U) \subseteq \bar{V}^{\circ}$. Let $y \in \bar{U}$. For any open set $W$ containing $f(y)$ there exists, by almost continuity of $f$, an open set $A$ containing $y$ such that $f(A) \subseteq \bar{W}^{\circ}$. Since $y \in \bar{U}$, we have $A \cap U \neq \varnothing$. Therefore, $\varnothing \neq f(A \cap U)$ $\subseteq \bar{V}^{\circ} \cap \bar{W}^{\circ} \subseteq \bar{W}$. Since $\bar{V}^{\circ} \cap \bar{W}^{\circ}$ is open we have $\bar{V}^{\circ} \cap \bar{W}^{\circ} \cap W \neq \varnothing$ : that is, $\bar{V}^{\circ} \cap W \neq \emptyset$. Since this is true for every open set containing $f(y)$ we have $f(y) \in \bar{V}$. Also since this is true for every $y \in \bar{U}$ we obtain $f(\bar{U}) \subseteq \bar{V}$ : that is, $f$ is closure continuous.

Recall that a subset $A$ of a space $X$ is called closure (almost) compact if every open cover of $A$ has a finite subcollection whose closures cover $A$. Closure compactness was introduced as $H(i)$-spaces in [8] as a generalization of absolutely closed ( $H$-closed) spaces in [1].

\footnotetext{
${ }^{\dagger}$ The author was supported by Birzeit University under grant 235-17-98-9.
}

Glasgow Math. J. 40 (1998) 263-264. 
Clearly every compact set is closure compact but not conversely as is shown in the next example.

EXAMPLE 1. Let $X$ be any uncountable space with the cocountable topology. Then every subset of $X$ is closure compact, but the only compact subsets of $X$ are the finite ones.

The next theorem shows that closure compactness is preserved under closure continuous functions.

ThEOREM 2. Let $f: X \rightarrow Y$ be closure continuous and let $K$ be a closure compact subset of $X$. Then $f(K)$ is a closure compact subset of $Y$.

Proof. Let $\mathcal{V}$ be an open cover of $f(K)$. For each $k \in K, f(k) \in V_{k}$ for some $V_{k} \in \mathcal{V}$. By closure continuity of $f$, there exists an open set $U_{k}$ containing $x$ such that $f\left(\bar{U}_{k}\right) \subseteq \bar{V}_{k}$. The collection $\left\{U_{k}: k \in K\right\}$ is an open cover of $K$ and so, since $K$ is closure compact, there is a finite subcollection $\left\{U_{k}: k \in K_{0}\right\}$, where $K_{0}$ is a finite subset of $K$, and $\left\{\bar{U}_{k}: k \in K_{0}\right\}$ covers $K$. Clearly $\left\{\bar{V}_{k}: k \in K_{0}\right\}$ covers $f(K)$ and thus $f(K)$ is a closure compact subset of $Y$.

COROllary 1. Let $f: X \rightarrow Y$ be almost continuous and let $K$ be a closure compact subset of $X$. Then $f(K)$ is a closure compact subset of $Y$.

As a consequence of the corollary, we get Theorem 3.3 and Lemmas 3.2, 3.3 in [10] and Theorem 3.4 in [4].

\title{
REFERENCES
}

1. P. Alexandroff and P. Urysohn, Mèmoire sur les espaces topologiques compacts, Verh. Nederl. Akad. Wetensch. Afd. Natuurk. Sect. I 14 (1929), 1-96.

2. D. R. Andrew and E. K. Whittlesy, Closure continuity, Amer. Math. Monthly 73 (1966), 758-759.

3. S. V. Fomin, Extensions of topological spaces, C. R. Dokl. Akad. Sci. URSS (M. S.) 32 (1941), 114-116.

4. L. Herrington, Properties of nearly-compact spaces, Proc. Amer. Math. Soc. 45 (1974), 431-436.

5. P. E. Long and E. E. McGehee, Properties of almost continuous functions, Proc. Amer. Math. Soc. 24 (1970), 175-180.

6. P. E. Long and D. A. Carnahan, Comparing almost continuous functions, Proc. Amer. Math. Soc. 38 (1973), 413-418.

7. T. Noire, On weakly continuous mappings, Proc. Amer. Math. Soc. 46(1) (1974), 120-124.

8. C. T. Scarborough and A. H. Stone, Products of nearly compact spaces, Trans. Amer. Math. Soc. 124 (1966), 131-147. $63-73$.

9. M. K. Singal and A. R. Singal, Almost continuous mappings, Yokohama Math. J. 16 (1968),

10. M. K. Singal and A. Mathur, On nearly compact spaces, Boll. Un. Mat. Ital. 4(2) (1969), 702-710.

\author{
Mathematics Department \\ BiRzEIT UNIVERSITY \\ PO Box 14 \\ BIRZEIT \\ West Bank \\ Palestine
}

